\title{
Study on the Performance of a Sirocco Fan (Flow Around the Runner Blade)
}

\author{
Tsutomu Adachi and Naohiro Sugita \\ Department of Mechanical Engineering for Transportation, Osaka Sangyo University, Osaka, Japan
}

Yousuke Yamada

Toshiba Corporation, Tokyo, Japan

Initially in this research, effects of blade inlet and outlet angles on the performance were considered by measuring performances. Twelve impellers with various blade angles were used for the measurements. The most suitable inlet and outlet angles and the inclination angle were acquired. Then, measurements on the inlet and outlet flows and their axial and circumferential distributions were taken for various operating conditions at the various measuring positions. The turning angles through the blade were calculated from these measured results. The inlet and outlet flows of the sirocco fan are not uniform around the circumference. The distributions of the flow have some relations with the development of flow in the inlet part and in the casing. Discussions were made on the flow to have a improved performance.

Keywords Siricco fan, Fan, Flow in turbomachinery, Flow measurement, Three dimensional flow

\section{INTRODUCTION}

A sirocco fan is a centrifugal fan with a forward curved blade. It is used for low pressure but with a large discharge use. The outlet port of this fan may have a rectangular shape and it is sometimes contained in the air conditioning apparatus. Indeed, a forward curved vane can give large angular momentum to the fluid, but it cannot make for better efficiency.

According to the measured results on the flow in the neighborhood of the outlet of a moving blade by Yamamoto et al. (1995) and Raj and Swim (1981), the uniform flow cannot be obtained

Received 25 June 2002; accepted 10 July 2002.

Address correspondence to Tsutomu Adachi, Department of Mechanical Engineering for Transportation, Osaka Sangyo University, 3-1-1, Nakagaito Daito, Osaka, 574-8530, Japan. E-mail: adachi@tm. osaka-sandai.ac.jp because of a sudden turning of the inlet flow. In the circumferential direction, the inlet flow develops its magnitude of velocity and its direction. However, its developing procedure and flow pattern in the casing is not clear yet. Yamazaki and Sato (1986) and Nakajima et al. (1988) found a large vortex on the shroud side. As the inlet flow into the forward curved vane of the centrifugal fan is the increasing flow, the occurrence of turbulence is sometimes suppressed as reported by Totsuka et al. (1996). Morinushi (1991) reports on the noise source arising from the separating flow. Researches have been done on the noise source arising from the separating flow, however, there is not much research on the optimum condition to design the sirocco fan with the best performance.

In this article, 12 impellers with various blade shapes were tested in the same casing. The measurements of the threedimensional steady and time-variant velocity at the inlet and outlet of the impeller were done using four impellers with better performances. The developing flow procedure in the circumferential and axial direction in the casing were shown. The relations between the blade shape with respect to the inlet and outlet flows and their performances were considered.

\section{EXPERIMENTAL APPARATUS AND RESULTS OF PERFORMANCE TESTS}

Experimental Apparatus and Method of the Measurements

Figure 1 and Table 1 show the impeller used for this research. The inner and outer diameters of these impellers are approximately 130 and $160 \mathrm{~mm}$. The number of blades is 36 , except for impeller I. The inclination angle $I_{\mathrm{r}}$ represents the angle which makes the line connecting the inlet and outlet of the blade with the radial line that goes through the inlet edge of the blade. The forward inclination takes the positive values and the backward inclination takes the negative values, respectively. To regulate the discharge, the opening of the damper placed at the end of the ducts was changed. The pressure difference 


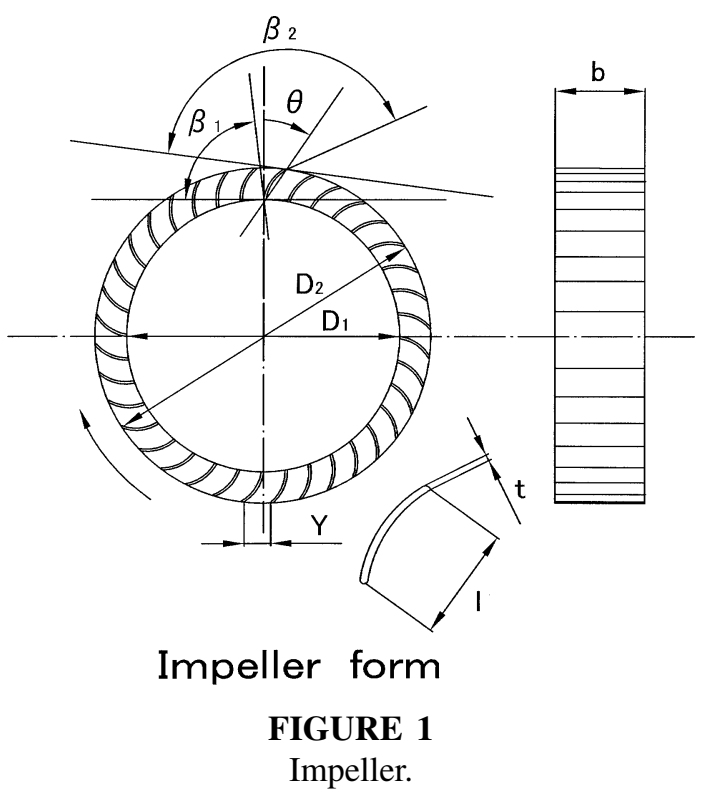

between the up and down side of the orifice plate, static pressure on the casing wall, and input electrical power were measured. These measurements were done for various rotational speeds, i.e., 800, 1200, 1600, 2000, 2400, 2800, and 3100 rpm, respectively.

\section{Performance Curves}

Figure 2 shows the performance curves and the efficiency curves. Impellers III and IV were designed considering a collision-free inlet to the runner blade from the velocity triangle. Indeed, the performance curves for these impellers show a comparatively good efficiency, yet they cannot get a large discharge. On the contrary, many impellers with a large inlet angle, i.e., near $90^{\circ}$, can get a large discharge. The best pressure, efficiency, and discharge performance was acquired for the impeller II. The effect of blade thickness on the performance is also important. Comparing performance curves of impellers II and II,' the inlet and outlet angles and the inclination angles are almost the same for these impellers. Impeller II can get more discharge and higher efficiency than impeller II'. The reason why these differences arise appears to come from the blade thickness.

The inclination angle $I_{\mathrm{r}}$ and the outlet angle $\beta_{2}$ are almost the same for impellers II' and X. Indeed, many differences cannot be seen in the efficiency, but the discharge is large for impeller X. This difference seems to come from the differences in the blade inlet angle. From these reasons, the best values for the inlet angle appears to be $90^{\circ}$. Comparing performance curves of the impellers III, III,' and IV, the difference in the performance comes from the inclination angle and the outlet angle. For a blade with a circular arc, the outlet angle can be decided if we define the inlet and inclination angles. Then the effect of the inclination angle is also important. Figure 3 shows the effects of the Reynolds number on the efficiency. The blade diameter and circumferential velocity at the tip were taken for the representative length and the velocity. According to our experiment, it becomes clear that the loss increase can be prevented, if we take the Reynolds number to be more than $\operatorname{Re}=2 \times 10^{5}$. In our experiments, this number was attained at $2800 \mathrm{rpm}$.

\section{VELOCITY DISTRIBUTIONS AT THE INLET TO THE IMPELLER}

Inlet velocity distribution measurements were done at the four circumferential positions using a five-hole probe. These measurements were done for the impellers II, II,' IX, and X. The operating conditions were at the design discharge and the maximum discharge. The measuring points were five points in the axial directions, including shroud and hub sides, as shown in Figure 4. The circumferential positions were four points and were selected to show the inlet flow variations in the circumferential direction.

TABLE 1

Dimension of Impellers

\begin{tabular}{|c|c|c|c|c|c|c|c|c|c|c|c|c|}
\hline & $D_{1}$ & $D_{2}$ & $D_{1} / D_{2}$ & $I$ & $b$ & $\theta$ & $\beta_{1}$ & $\beta_{2}$ & $I_{\mathrm{r}}$ & $t$ & $Y$ & $Z$ \\
\hline I & 139.0 & 159.9 & 0.87 & 13.4 & 49.3 & 44.2 & 103.8 & 154.5 & 0.43 & 1.1 & 11.3 & 38 \\
\hline II & 133.5 & 159.3 & 0.84 & 14.5 & 47.7 & 31.4 & 77.5 & 156.3 & 0.41 & 1.0 & 12.9 & \\
\hline III & 124.0 & 156.1 & 0.79 & 17.2 & 46.3 & -24.5 & 14.4 & 121.9 & 1.71 & & 10.5 & \\
\hline IV & 128.2 & 160.4 & 0.80 & 16.5 & 46.4 & -13.7 & 11.6 & 143.9 & 1.18 & 2.0 & 10.6 & \\
\hline V & 133.5 & 163.6 & 0.82 & 15.9 & 46.0 & 20.3 & 83.8 & 133.0 & 0.24 & & 10.9 & \\
\hline VI & 128.9 & 163.4 & 0.79 & 19.8 & 46.4 & 33.2 & 86.4 & 152.4 & 0.38 & 2.4 & 10.6 & \\
\hline VII & 129.9 & 161.6 & 0.80 & 17.4 & 46.1 & 27.3 & 92.9 & 136.1 & 0.29 & 2.3 & 10.7 & 36 \\
\hline VIII & 131.0 & 160.0 & 0.82 & 17.1 & 46.4 & 35.7 & 89.4 & 154.8 & 0.40 & 2.4 & 10.3 & \\
\hline IX & 130.1 & 160.1 & 0.81 & 19.6 & 46.2 & 45.5 & 93.1 & 167.9 & 0.49 & 2.1 & 10.6 & \\
\hline X & 128.8 & 160.4 & 0.80 & 18.6 & 46.6 & 38.0 & 87.0 & 160.7 & 0.44 & & 12.0 & \\
\hline $\mathrm{II}^{\prime}$ & 130.1 & 163.6 & 0.80 & 18.6 & 46.0 & 32.2 & 77.9 & 160.7 & 0.41 & 2.0 & 12.3 & \\
\hline IIII & 127.0 & 160.2 & 0.79 & 16.1 & 46.2 & -8.1 & 8.7 & 156.8 & 0.94 & & 12.0 & \\
\hline
\end{tabular}



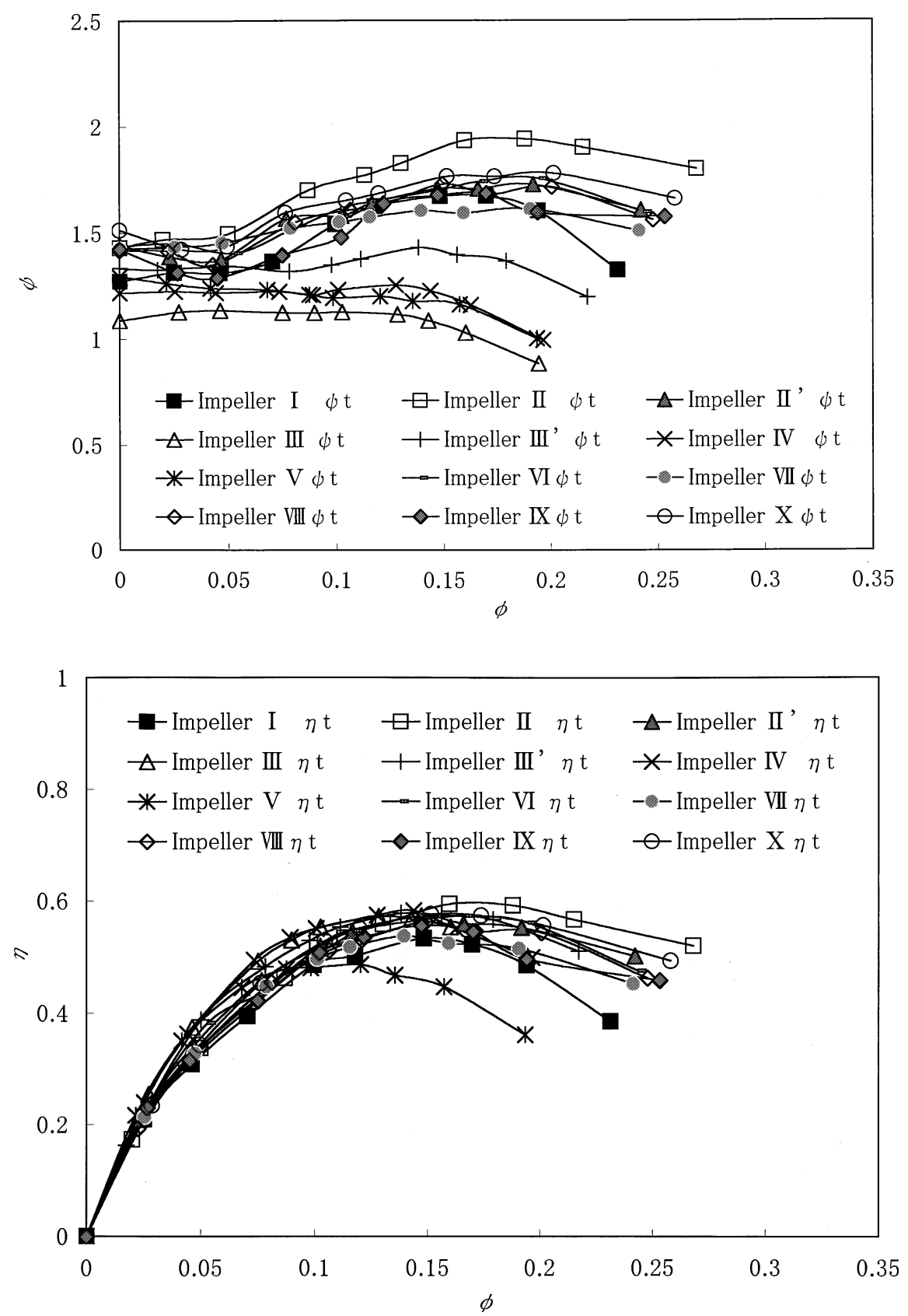

FIGURE 2

Performance curves.

\section{Relative Inlet Velocity Distributions at the Inlet of the Impeller}

Figure 5 shows an example of the relative inlet velocity distribution at the shroud side of the impeller II. As shown in this figure, the inlet velocity flows in the counter direction especially at $\theta_{\mathrm{c}}=15^{\circ}$, i.e., near the volute tongue. There is scarcely any flow velocity at $\theta_{\mathrm{c}}=105^{\circ}$ and $195^{\circ}$. The inlet velocity takes a positive value at $\theta_{\mathrm{c}}=285^{\circ}$. The relative velocity takes the positive larger values, if the measuring point becomes near the hub. It also takes the positive larger values with an increase in the circumferential angle.

Figure 6 shows the variations of the relative inlet velocity distribution with the distance from the shroud to the hub. The distributions are almost the same for the 4 impellers. The velocities take the smaller values at the shroud side and take the larger values with a distance from the shroud to the hub. The main flow region is near the hub at $\theta_{\mathrm{c}}=15 \sim 195^{\circ}$ but the distributions are almost uniform at $\theta_{\mathrm{c}}=285^{\circ}$. 


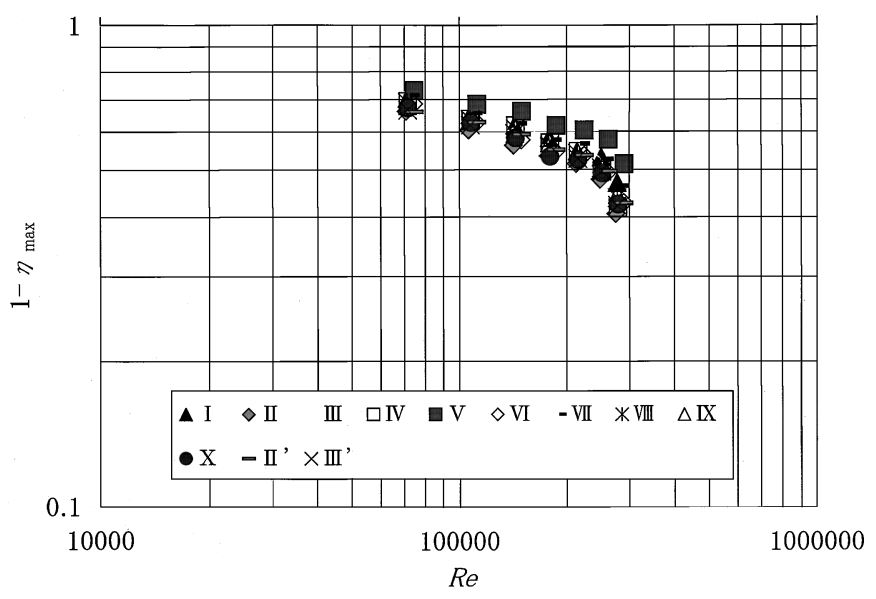

FIGURE 3

Effects of Reynolds number on the efficiency.

\section{The Absolute Velocity Distribution at the Inlet to the Impeller}

Figures 7, 8, and 9 show the radial, axial and circumferential components of absolute inlet velocity distributions with axial distance for the four impellers. The radial components (Figure 7) show counter flow especially at $\theta_{\mathrm{c}}=15^{\circ}$ and $105^{\circ}$. It seems to be the effect of the volute tongue. At $\theta_{c}=285^{\circ}$ the effects of the tongue can scarcely be recognized. The flow velocity is almost uniform along the blade width. Figure 8 shows the distribution of the axial component. In the figure the ordinate, the plus value shows the direction of flow to the hub from the shroud. For $\theta_{\mathrm{c}}=15^{\circ}, 105^{\circ}$, and $195^{\circ}$, the axial components are almost uniform from the shroud to the hub. But at $\theta_{\mathrm{c}}=285^{\circ}$ the flow directs towards hub at the shroud and towards shroud at the hub. It shows that a vortex develops at the entrance as stated

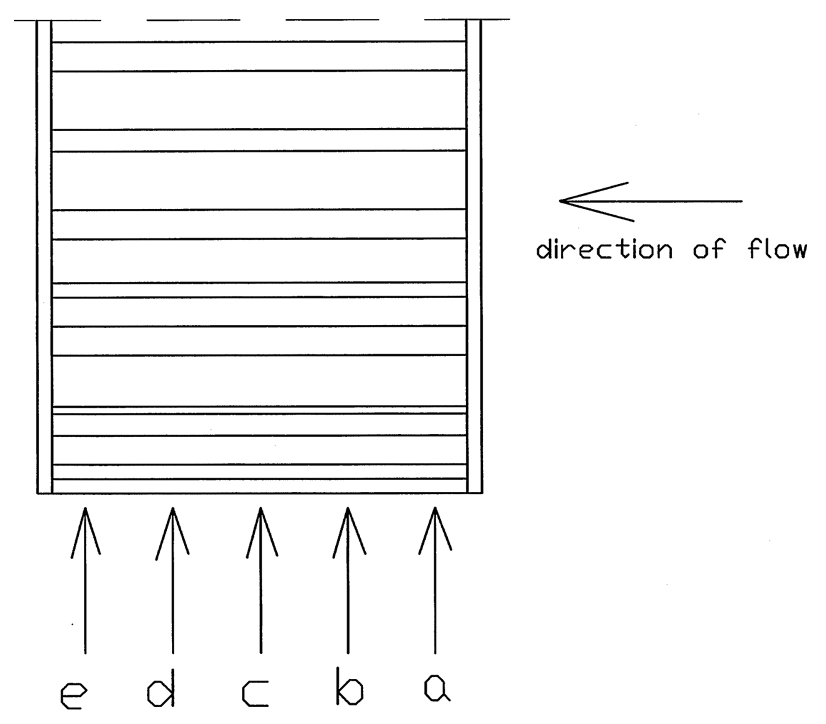

FIGURE 4

Flow velocity measuring points.

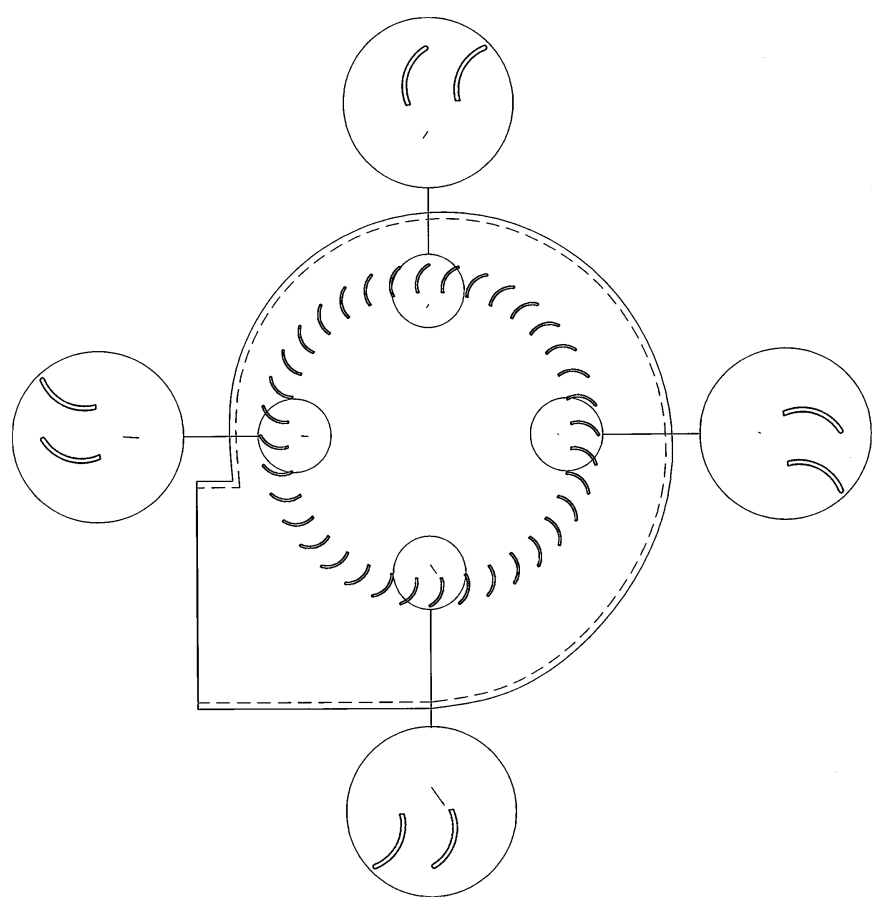

FIGURE 5

Relative inlet velocity distribution at the shroud side (point a) for impeller II.

by Yamazaki and Sato (1986). Figure 9 shows the component in the circumferential direction. The circumferential velocity components is large at $\theta_{\mathrm{c}}=15^{\circ}$, especially on the hub. They decrease in the axial and circumferential directions. It is because that the flow is suppressed by the volute tongue.

\section{Incidence Angle}

The distribution of incidence angle defined by $i=\beta_{1}-\gamma_{1}$ is shown in Figure 10. For these four impellers the tendency is almost the same. The incidence angle takes a large values at the shroud for $\theta_{\mathrm{c}}=15^{\circ}, 105^{\circ}$, and $195^{\circ}$. It shows that the flow goes backward.

\section{THE VELOCITY DISTRIBUTIONS AT THE OUTLET OF THE IMPELLER}

\section{Experimental Apparatus and Method}

A hot wire probe, as shown in Figure 11, was used to measure velocity distributions at the outlet from the impeller blade. Tungsten wire with $4 \mu \mathrm{m}$ thickness and $1 \mathrm{~mm}$ length is attached with a $45^{\circ}$ angle. The inserting part of the probe is also shown in the same figure. The hot wire probe can be inserted in four various ways. To measure velocity and its direction, measurements were made four times at the same positions. Then the probe axis was directed to the angle $\left(\pi-\beta_{2}\right)$ with the circumferential direction. The distance of the tungsten wire and the outer surface of the impeller was $0.5 \mathrm{~mm}$ as shown in Figure12. The number of revolutions was set to $800 \mathrm{rpm}$. It was set to lower numbers of 


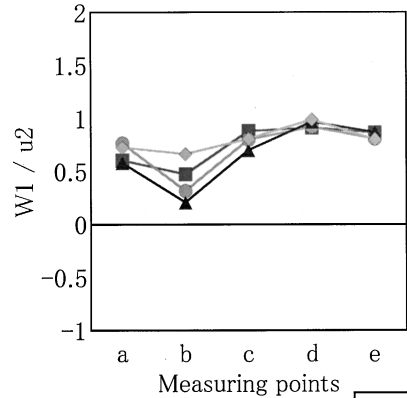

i) $\theta \mathrm{c}=15^{\circ}$

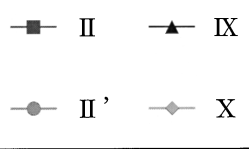

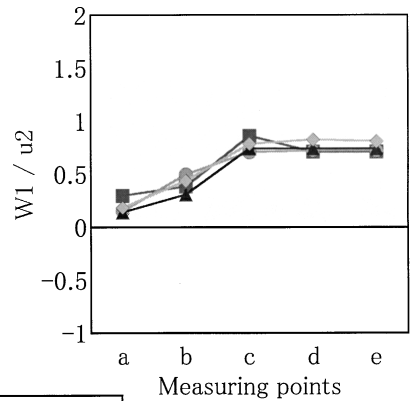

ii) $\theta \mathrm{c}=105^{\circ}$

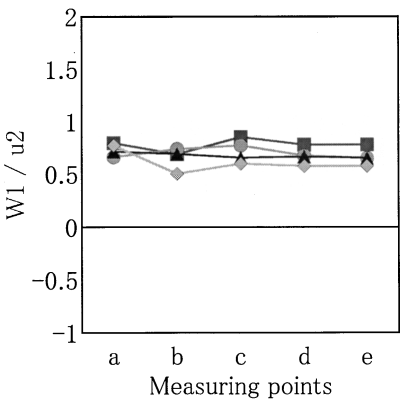

iv) $\theta \mathrm{c}=285^{\circ}$

FIGURE 6

Variations of relative inlet velocity distribution at inlet of impeller.

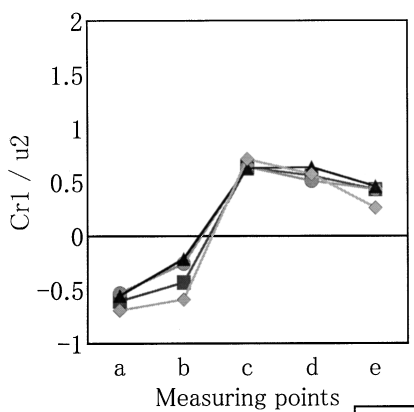

i ) $\theta \mathrm{c}=15^{\circ}$

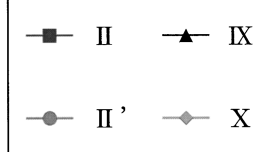

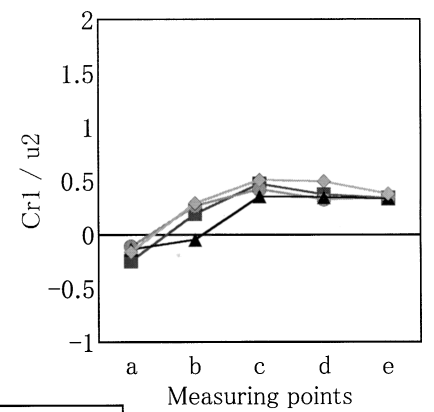

ii) $\theta \mathrm{c}=105^{\circ}$

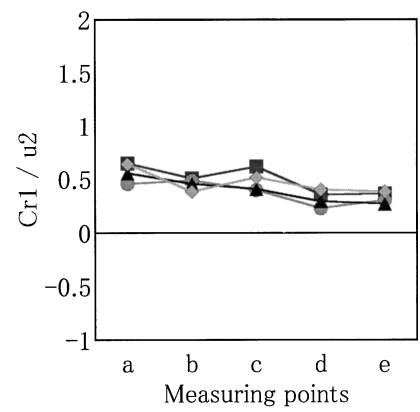

iv) $\theta \mathrm{c}=285^{\circ}$

iii) $\theta \mathrm{c}=195^{\circ}$

FIGURE 7

Radial components of absolute inlet velocity distribution with axial distance. 


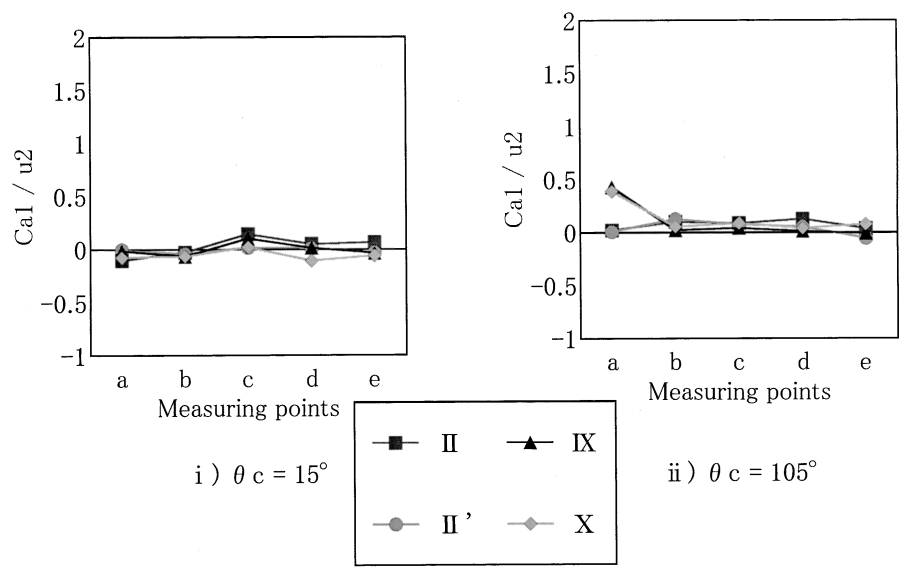

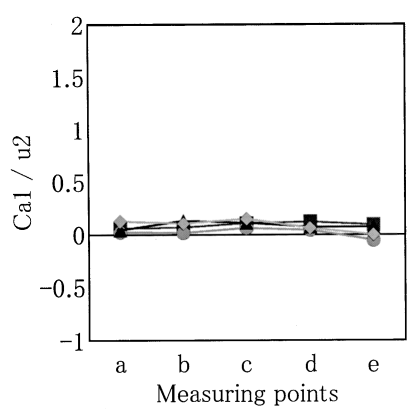

iii) $\theta \mathrm{c}=195^{\circ}$

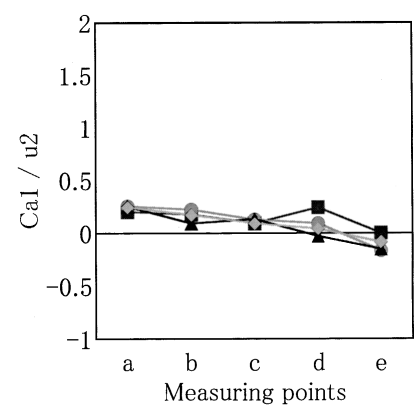

iv) $\theta \mathrm{c}=285^{\circ}$

FIGURE 8

Axial components of inlet velocity distribution with axial distance.

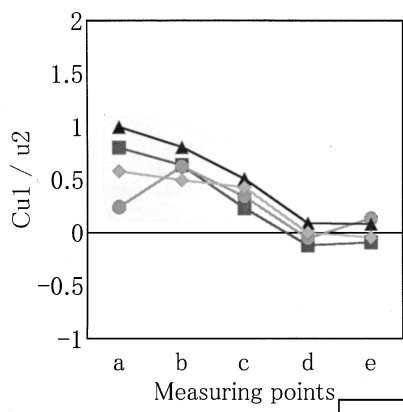

i) $\theta \mathrm{c}=15^{\circ}$
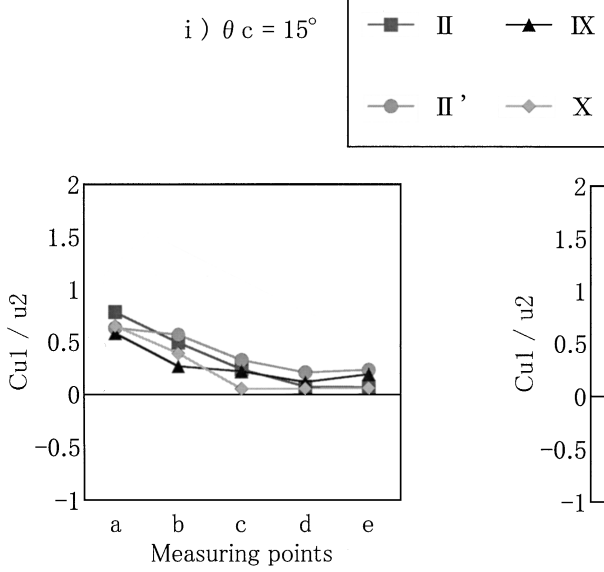

iii) $\theta \mathrm{c}=195^{\circ}$

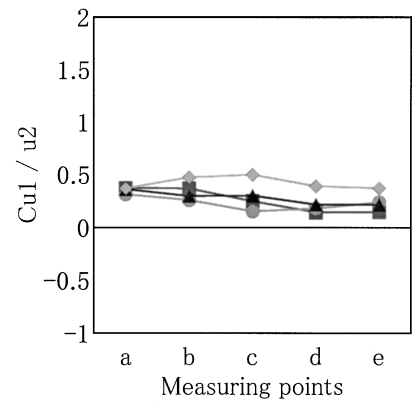

iv) $\theta \mathrm{c}=285^{\circ}$

FIGURE 9

Circumferential components of inlet velocity distribution with axial distance. 


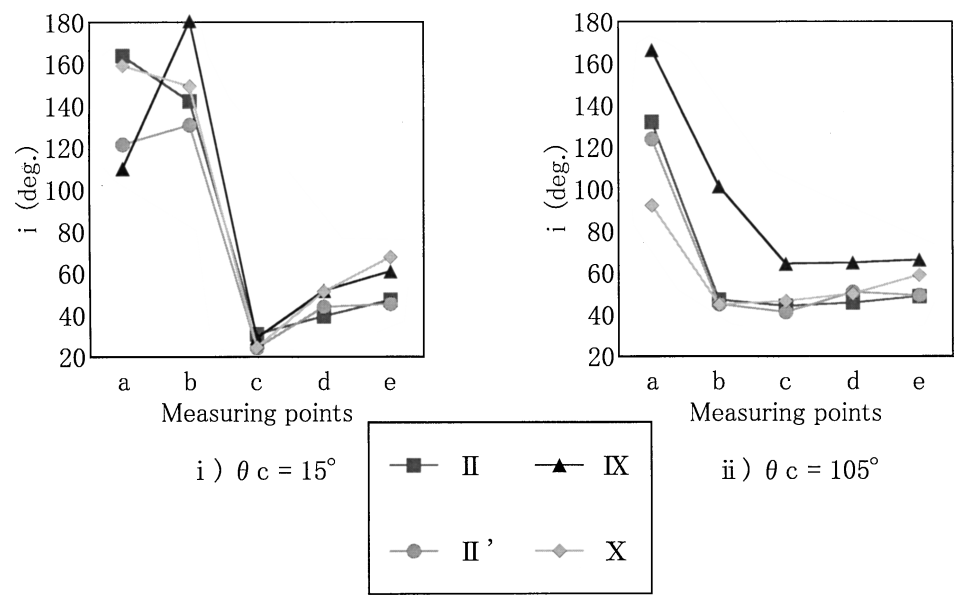

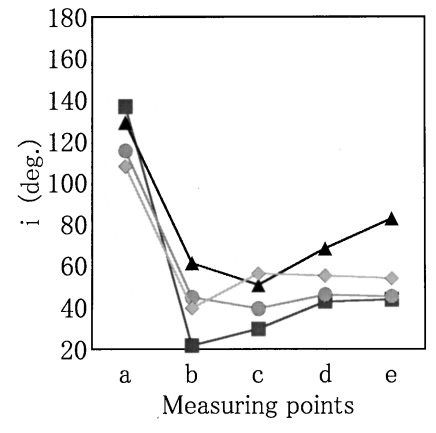

iii) $\theta \mathrm{c}=195^{\circ}$

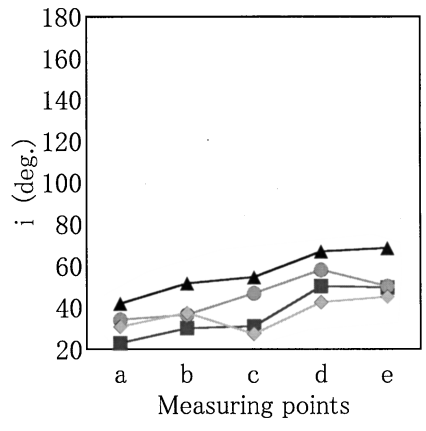

iv) $\theta \mathrm{c}=285^{\circ}$

FIGURE 10

Distribution of incidence angle $i=\beta_{1}-\gamma_{1}$ with axial distance.

revolutions to avoid the damage from vibration. The probe was traversed in the axial direction and measurements were done at five points with equal distances apart from the shroud to the hub as shown in Figure 4, i.e., a, b, c, d, e. Sampling was started by the signal when the fixed position was at the fixed distance of one blade pitch. The sampling period was set to collect data on four positions at an equal distance of one blade pitch. Every data was summed up $2^{8}$ times and the averaged time value was measured. Then the random components disappeared from the

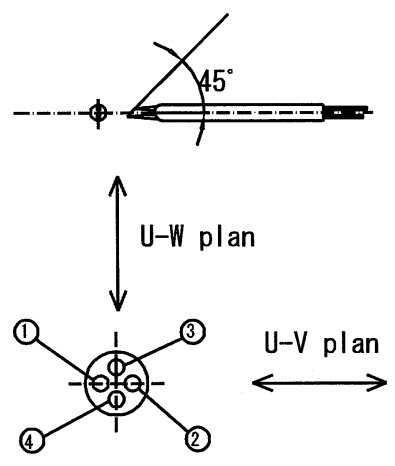

FIGURE 11

Hot wire probe. (1) impeller; (2) hot wire probe.

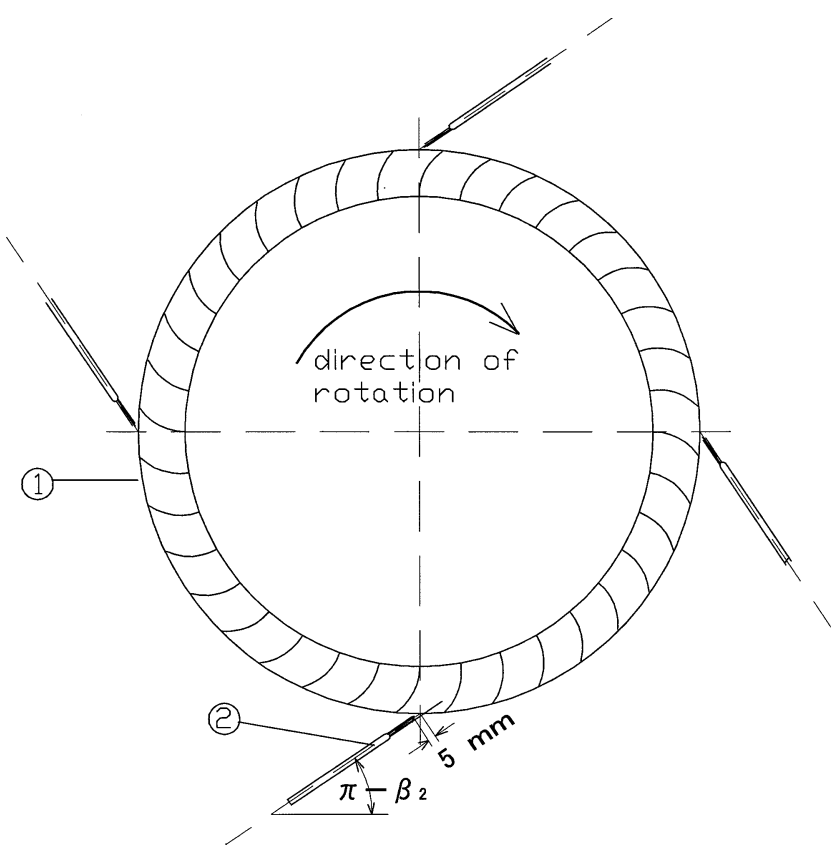

FIGURE 12

Positions of hot wire probe at the four circumferential positions. 


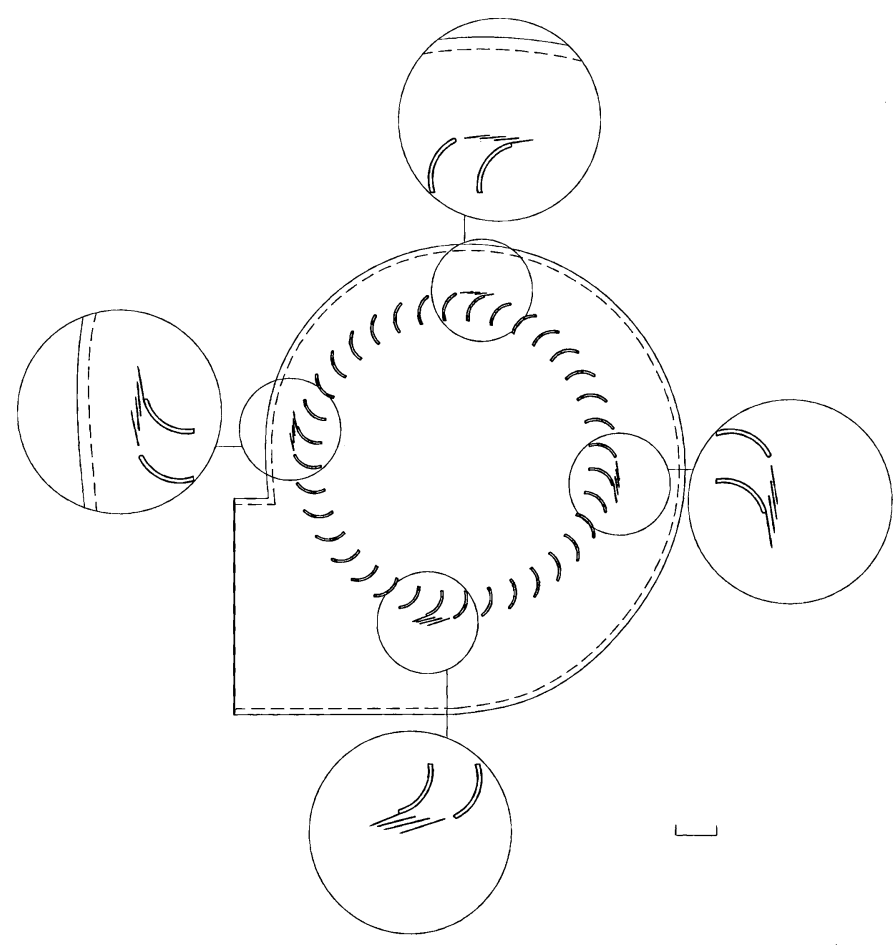

FIGURE 13

Relative velocity distribution at the outlet of impeller II for point $a$, at $\phi_{\text {des }}$.

signal. The magnitude of velocity and its direction were calculated from these four signals taken at four various measuring holes and at the same measuring point. To show the measured results two planes were taken; one is the U-V plane, i.e., the plane including the fan axis, and the other is the U-W plane, i.e., the meridian plane. Observations of the flow were made at the design discharge and at the maximum discharge condition. Outlet flow velocity was also measured using four impellers: II, II,' IX, and X .

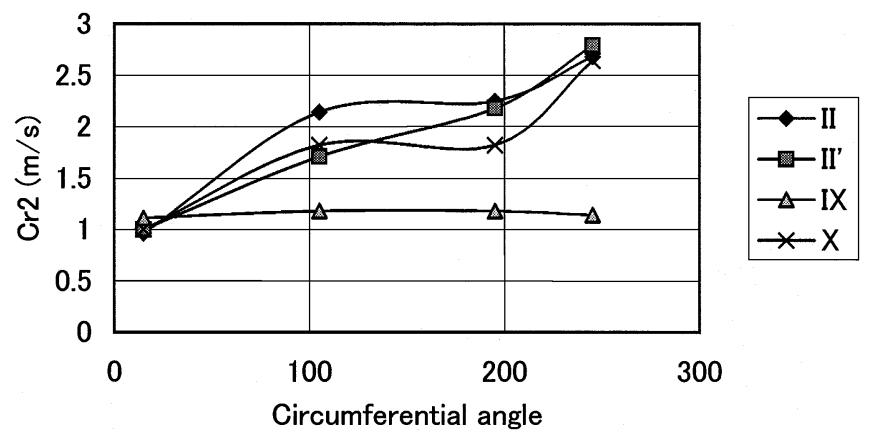

FIGURE 14

Radial components of velocity distribution with circumferential direction at $\phi_{\max }$.

\section{Relative Velocity Distribution at the Outlet of the Impeller}

Figure 13 shows relative velocity distribution at the outlet of the impeller II. At $\theta_{\mathrm{c}}=15^{\circ}$, the outlet velocity directs to the circumferential direction and their magnitudes are larger at SS and smaller at PS. It shows the effect of the tongue as stated by Cau et al. (1987). With an increase in $\theta \mathrm{c}$, the flow velocity take larger values at PS and becomes smaller at SS. The flow separates at the leading edge of the SS and reattaches at the trailing edge again. Then the flow flows along the suction surface for impellers II, II,' and X. For the impeller IX, the blade inclination angle and the blade outlet angle take larger values, and the flow passage turn suddenly, then a secondary flow arise in the passage. The flow velocity takes a large value and flow attaches the impeller outside. Figure 14 shows variations of the absolute radial velocity component with circumferential direction for the four impellers. From this figure, radial absolute velocity component increases with an increase in the circumferential distance for impellers II, II,' and X. This shows favorable increase in flow discharge along the impeller. But for the impeller IX, this value is small and does not increase in the circumferential direction. This shows that the blade outlet angle is too large.

\section{The Deviation Angle}

The deviation angle defined by

$$
\delta=\beta_{2}-\gamma_{2}
$$

is plotted in Figure 15. At $\theta_{\mathrm{c}}=15^{\circ}$, all the data take minus values for all impellers measured. This means that the flow circulates around the impeller. With an increase in $\theta \mathrm{c}$, the deviation angle increases. It takes a lesser value at SS and increases gradually across the passage to the PS. The flow attaches along the impeller outside at SS, but the radial component of velocity increases while it flows along the blade. The SS surface corresponds to the wake region, and the PS surface to the jet region, respectively, at $\theta_{\mathrm{c}}=285^{\circ}$. From the measured results at the inlet to the impeller, the once separated flow through the impeller flows out along the blade. It arises because the flow is increasing.

\section{Flow in the Outlet Surface of Impeller}

Figure 16 shows the flow in the outlet surface of impeller II at the design discharge. In these figures the frame shows one pitch blade outlet surface. The left side shows shroud and the right side shows hub; the upper side shows SS and the lower side shows PS. The flow at $\theta_{\mathrm{c}}=15^{\circ}$ directs the left side at the shroud and the right side at the hub. It shows as if the flow is suppressed by the tongue. The flow appearance changes in the circumferential direction. It directs to the hub side at the shroud and it seems that there is a large inlet vortex and it develops in the circumferential direction $\theta$ as stated by Yamazaki and Sato (1986). 


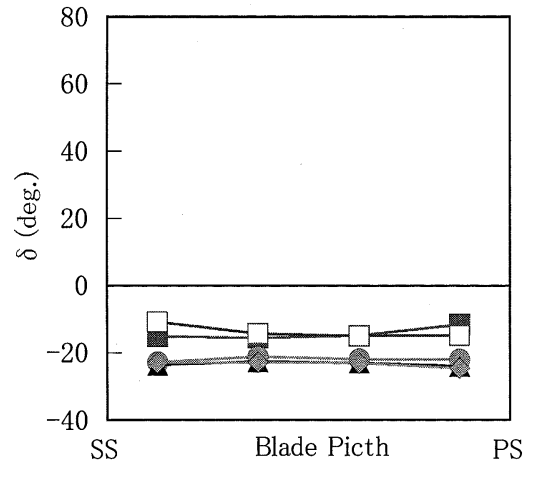

i) $\theta \mathrm{c}=15^{\circ}$

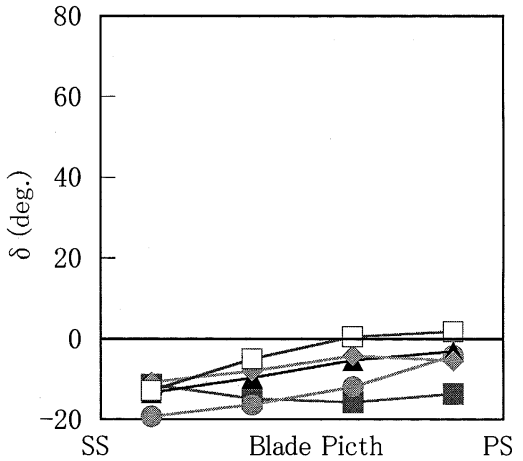

ii) $\theta \mathrm{c}=105^{\circ}$

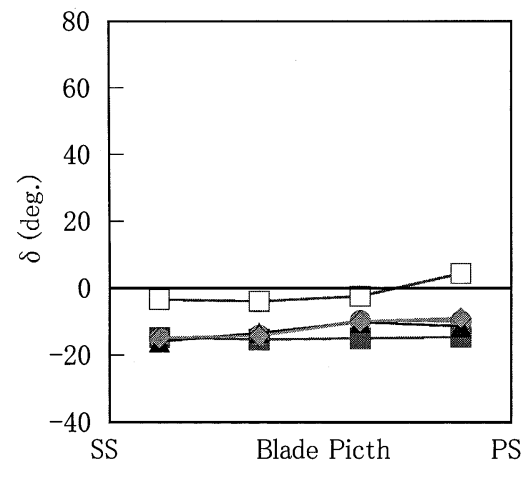

iv) $\theta \mathrm{c}=195^{\circ}$

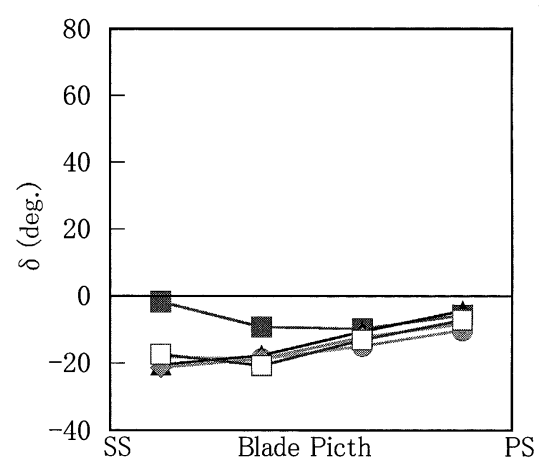

iii) $\theta \mathrm{c}=285^{\circ}$

\section{Impeller II, $\phi$ des}

FIGURE 15

Deviation angle $\delta=\beta_{2}-\gamma_{2}$ distribution for impeller II, at $\phi_{\text {des }}$.

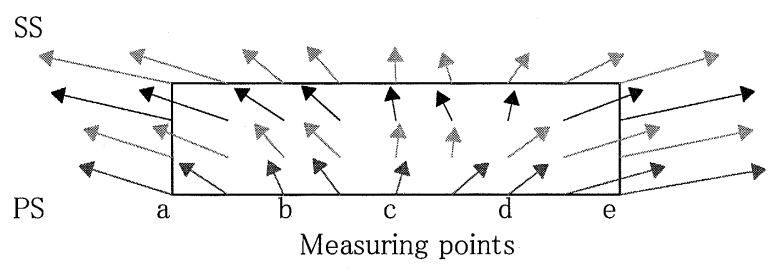

i ) $\theta \mathrm{c}=15^{\circ}$

SS

PS

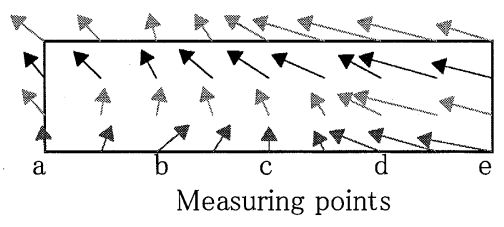

iii) $\theta \mathrm{c}=195^{\circ}$
SS

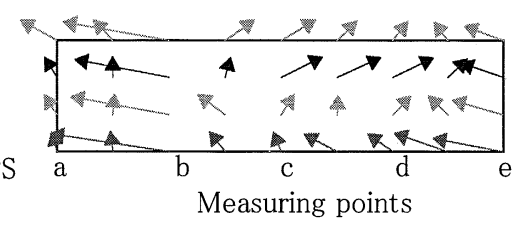

ii) $\theta \mathrm{c}=105^{\circ}$

SS
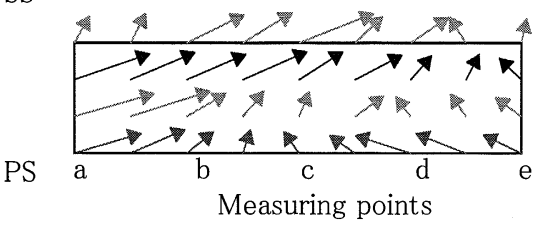

iv) $\theta \mathrm{c}=285^{\circ}$

FIGURE 16

Flow in the outlet surface for impeller II, at $\phi_{\text {des }}$. 


\section{CONCLUSIONS}

In this article, 12 impellers with various blade shapes were tested in the same casing. From the results of the performance test the favorable blade inlet and outlet angles were discussed. Then measurements of the inlet and outlet flow were measured using four impellers with better performances. The steady and time-variant three-dimensional flow in the axial and circumferential distributions were measured. From these results, the following results were obtained.

1. The best inlet and outlet angles are $75 \sim 90^{\circ}$ and $150 \sim 160^{\circ}$, respectively. The inlet angle, decided from the velocity triangle, is too small to obtain a large discharge. The outlet flow circulates around the impeller for the outlet angle exceeding $160^{\circ}$.

2. The inlet flow flows in the counter direction near the volute tongue. The velocity takes the smaller value at the shroud side and the larger values with a distance from the shroud to the hub at $\theta_{\mathrm{c}}=105^{\circ}$ and $195^{\circ}$. The velocity takes uniform values with respect to the axial direction near $\theta_{c}=285^{\circ}$. The incidence angle takes a large values at the shroud for $\theta_{\mathrm{c}}=15^{\circ}, 105^{\circ}$, and $195^{\circ}$. It shows the counter flow.

3. The outlet flow directs to the circumferential direction and their magnitude are larger at SS and smaller at PS. With an increase in $\theta \mathrm{c}$, the flow velocity takes a larger value at PS and becomes smaller at SS. The flow separates at the leading edge of the SS and reattaches at the trailing edge again. Then flow flows along the suction surface. At $\theta_{\mathrm{c}}=15^{\circ}$, the deviation angle takes minus value. It shows the flow circulate around the impeller. With an increase in $\theta_{\mathrm{c}}$, deviation angle increases. The suppression of flow near the volute tongue can also be recognized in the flow of the impeller outlet surface.

\section{NOMENCLATURE}

$b \quad$ impeller width

$D_{1} \quad$ inner diameter of impeller

$\mathrm{D}_{2} \quad$ outer diameter of impeller

$l \quad$ blade chord

$i=\beta_{1}-\gamma_{1} \quad$ incidence angle

$I_{\mathrm{r}} \quad$ blade inclination angle

$t$

thickness of blade

$\begin{array}{ll}Y & \text { blade pitch } \\ Z & \text { number of blades } \\ \beta_{1} & \text { blade inlet angle } \\ \beta_{2} & \text { blade outlet angle } \\ \theta_{\mathrm{c}} & \text { circumferential angle measured from the volute } \\ \delta=\beta_{2}-\gamma_{2} & \text { tongue } \\ \phi & \text { deviation angle } \\ \psi & \text { pressure coefficient } \\ \eta & \text { efficiency of fan } \\ \text { SS } & \text { suction surface of blade } \\ \text { PS } & \text { pressure surface of blade }\end{array}$

\section{Subscripts}

$1 \quad$ inlet of blade

2 outlet of blade

s static pressure

d dynamic pressure

t total pressure

max. maximum value

\section{REFERENCES}

Cau, G., Mandas, N., Manfrida, and Nurzia, F. 1987. Measurements of primary and secondary flows in an industrial forward-curved centrifugal fan. Transactions of the ASME, Journal of Fluid Engineering 109:353-358.

Morinushi, K. 1991. Noise source of a multiblade fan. Transactions of the JSME, Ser. B (in Japanese) 57(543):3837-3844.

Nakajima, K. et al. 1988. A study on the improvement of performance of multi-blade fan. Turbomachinery (in Japanese), 16(12):677-683.

Raj, D., and Swim, W. B. 1981. Measurement of the mean flow velocity fluctuations at the exit of an FC centrifugal fan rotor. Transactions of the ASME, Journal of Engineering for Power 103(2):393-399.

Totsuka, J. et al. 1996. Flow instabilities observed in sirocco fan impeller. Transaction of the JSME, Ser. B, (in Japanese) 62(594):684691.

Yamamoto, K. et al. 1995. Measurements of velocity distribution in impellers of multi-blade fans bared on flow visualization. Turbomachinery (in Japanese), 23(10):604-609.

Yamazaki, S., and Sato, R. 1986. An Experimental study on the aerodynamic performance of multi-blade blowers (1st report, measurement of flow patterns within blowers). Transactions of the JSME, Ser. B (in Japanese) 52(484):3987-3992. 

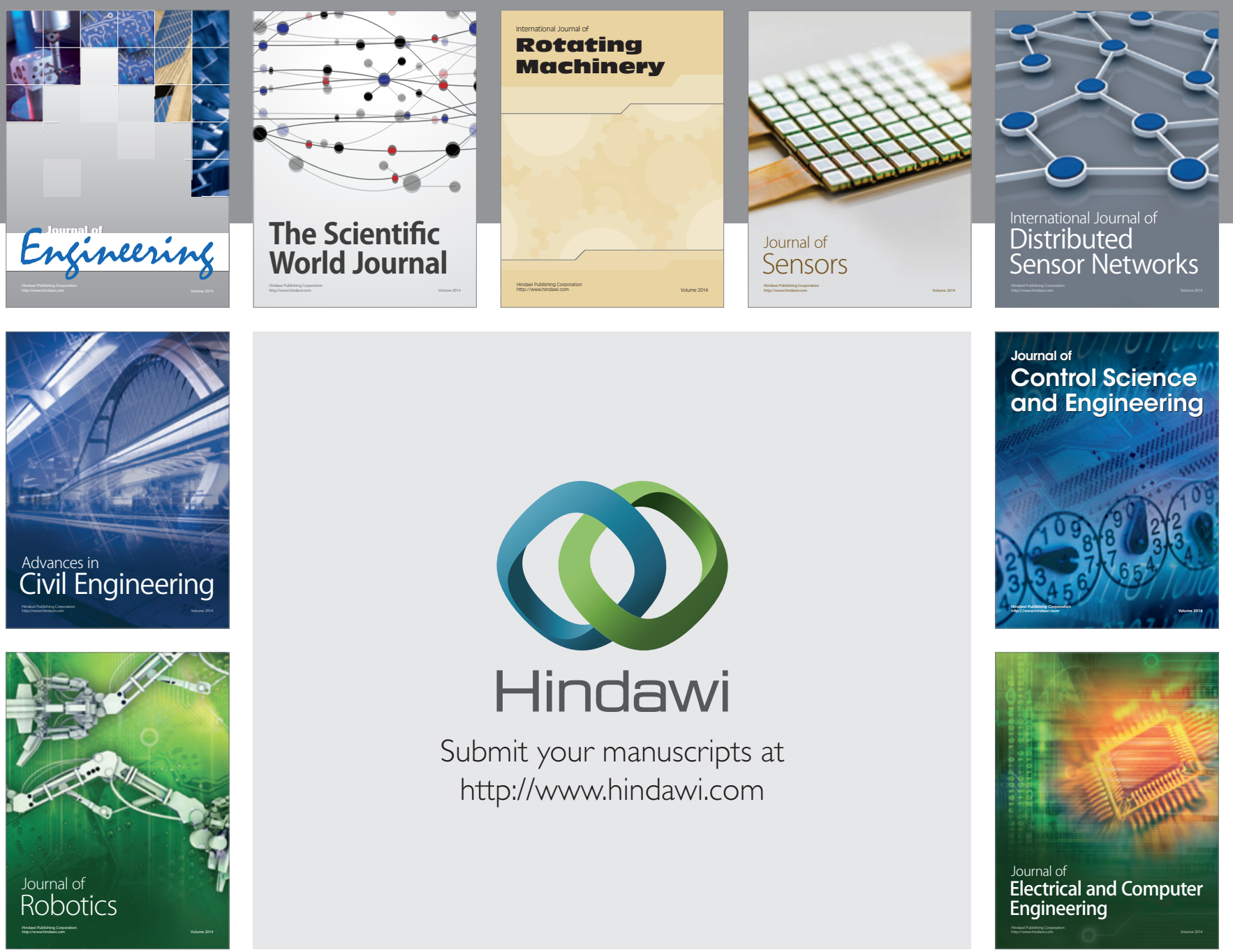

Submit your manuscripts at

http://www.hindawi.com
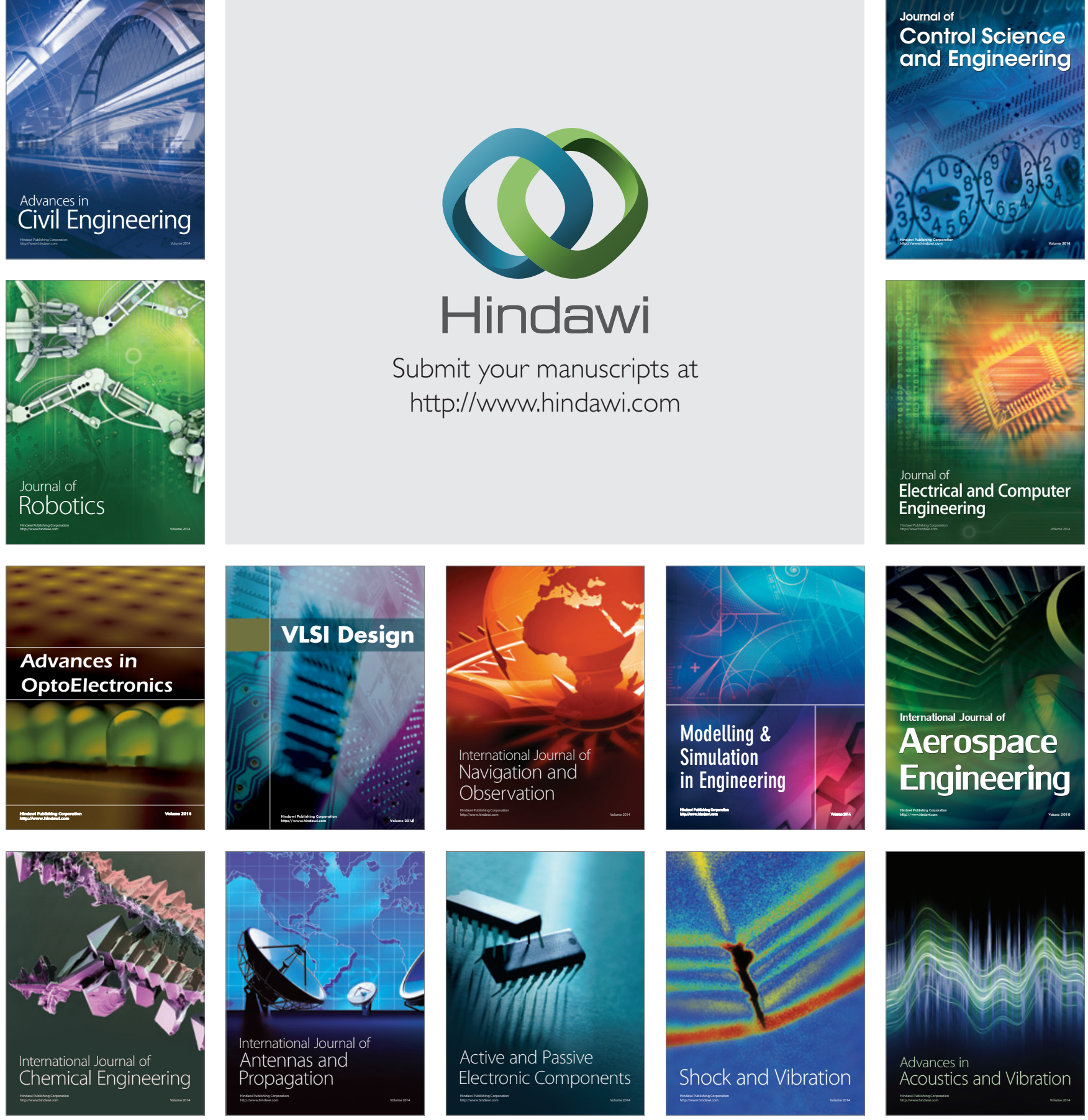